\title{
An efficient algorithm for the density Turán problem of some unicyclic graphs
}

\author{
Halina Bielak \\ Institute of Mathematics \\ Maria Curie Skłodowska University \\ Pl. M. Curie-Skłodowskiej 5 \\ 20-031 Lublin, Poland \\ Email: hbiel@hektor.umcs.lublin.pl
}

\author{
Kamil Powroźnik \\ Institute of Mathematics \\ Maria Curie Skłodowska University \\ Pl. M. Curie-Skłodowskiej 5 \\ 20-031 Lublin, Poland \\ Email: kamil.pawel.powroznik@gmail.com
}

\begin{abstract}
Let $H=(V(H), E(H))$ be a simple connected graph of order $n$ with the vertex set $V(H)$ and the edge set $E(H)$. We consider a blow-up graph $G[H]$.

We are interested in the following problem. We have to decide whether there exists a blow-up graph $G[H]$, with edge densities satisfying special conditions (homogeneous or inhomogeneous), such that the graph $H$ does not appear in a blow-up graph as a transversal.

We study this problem for unicyclic graphs $H$ with the cycle $C_{3}$. We show an efficient algorithm to decide whether a given set of edge densities ensures the existence of $H$ in the blow-up graph $G[H]$.
\end{abstract}

Index Terms-blow-up graph; density; Turán density problem; unicyclic graph.

\section{INTRODUCTION}

$\mathbf{T}$ URÁN [10] stated the first results in extremal graph theory. Then many authors extended this subject and formulated similar and new Turán density problems. [1], [3], [4], [6], [8], [9] and [11] obtained interesting results for some families of graphs.

In this paper we present an algorithm for testing whether a unicyclic graph with a given set of edge densities is a factor (transversal) of a blow-up graph. Our algorithm has the time complexity at most $\mathcal{O}\left(n^{2}\right)$, where $n$ is the number of vertices of the unicyclic graph.

Csikvári and Nagy [5] discovered some interesting algorithm for testing whether a tree with a given set of edge densities is a factor of a blow-up graph. We extend their algorithm to the family of unicyclic graphs with the cycle $C_{3}$.

Now we define some notions and notations. Other definitions one can find in [2] and [7].

Let $H=(V(H), E(H))$ be a simple connected graph of order $n$ with the vertex set $V(H)$ and the edge set $E(H)$. By $P_{k}$ we denote the path with $k$ vertices. By $C_{k}$ we denote the cycle with $k$ vertices. The set $S \subset V(H)$ is called an independent vertex set if the subgraph of $H$ induced by $S$ has empty set of edges.

Let

$$
N_{H}(v)=\{x \in V(H) \mid\{v, x\} \in E(H)\}
$$

be the neighbourhood of the vertex $v \in V(H)$ in the graph $H$. $\left|N_{H}(v)\right|$ is called the degree of $v$ in $V(H)$. Each vertex of degree 1 in a graph $H$ is called a leaf of the graph $H$.

We say that the graph $H$ is r-regular if each vertex of $H$ has degree $r$. The set $M \subset E(H)$ is called the matching (or independent edge set) in the graph $H$ if the subgraph of $H$ induced by $M$ is 1-regular.

For a connected graph $H$ we define a blow-up graph $G[H]$ of the graph $H$ as follows. First we replace each vertex $i \in$ $V(H)$ by an independent set of vertices $A_{i}$. Throughout this paper $A_{i}$ is called a cluster. Next we connect vertices between the clusters $A_{i}$ and $A_{j}$ if $i$ and $j$ are adjacent in $H, i, j \in$ $V(H)$. The graph induced by $A_{i} \cup A_{j}$ in $G[H]$ is a subgraph of a complete bipartite graph. See Fig. 2 and Fig. 3 which present examples of a blow-up graphs $G[H]$ of the graph $H$ presented in Fig. 1.

For any two clusters we define the density between them by the following formula

$$
d\left(A_{i}, A_{j}\right)=\frac{e\left(A_{i}, A_{j}\right)}{\left|A_{i}\right|\left|A_{j}\right|},
$$

where $e\left(A_{i}, A_{j}\right)$ denotes the number of edges between the clusters $A_{i}$ and $A_{j}$.

The graph $H$ is a transversal of $G[H]$ if $\mathrm{H}$ is a subgraph of $G[H]$ such that we have a homomorphism

$$
\phi: V(H) \rightarrow V(G[H])
$$

for which $\phi(i) \in A_{i}$ for all $i \in V(H)$. Other terminology: $H$ is a factor of $G[H]$. An edge $e=\{i, j\}$ of the graph $H$ we denote by $e=i j$.

The density Turán problem can be defined as follows. Let us determine the critical edge density, denoted by $d_{\text {crit }}$, which ensures the existence of the subgraph $H$ of $G[H]$ as a transversal. Precisely, assume that all edges $e=\{i, j\}$ in the graph $H$ satisfy the condition

$$
d\left(A_{i}, A j\right)>d_{\text {crit }},
$$

where $i, j \in V(H)$. Then, no matter how we construct the blow-up graph $G[H]$, it contains the graph $H$ as a transversal. 


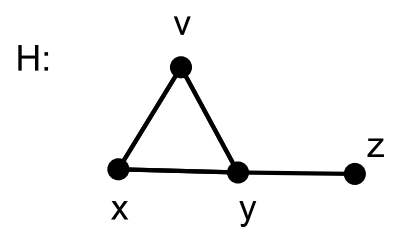

Fig. 1. The graph $H \in \mathcal{U}_{n, 3}$ with the vertex set $V(H)=\{x, y, z, v\}$.

On the other words, for any value $d<d_{\text {crit }}$ there exists a blow-up graph $G[H]$ such that

$$
d\left(A_{i}, A_{j}\right)>d
$$

for all edges $i j \in E(H)$ and it does not contain $H$ as a transversal. This problem was studied in [9].

By [5] we know that it is useful to consider more general problem. Let us assume that for every edge $e \in E(H)$ a density $\gamma_{e}$ is given. Now our task is to decide if the set of densities $\left\{\gamma_{e}\right\}_{e \in E(H)}$ ensure the existence of the graph $H$ as a transversal or we can construct a blow-up graph $G[H]$ such that

$$
d\left(A_{i}, A_{j}\right) \geq \gamma_{i j},
$$

but it does not induce the graph $H$ as a transversal. This more general setting allows to use inductive proofs (see the proof of Theorem 7). We call this general case as the inhomogeneous condition on the edge densities, while the above condition of having a common lower bound $d_{c r i t}(H)$ for densities is called the homogeneous case.

Let $\mathcal{U}_{n, p}$ be a family of unicyclic graphs of order $n$ with the cycle $C_{p}$. The path $P_{2}$ and the cycle $C_{3}$ are trivial unicyclic graphs for further considerations. In this paper we study the inhomogeneous density Turán problem for unicyclic graphs in the family $\mathcal{U}_{n, 3}$, i.e. with the unique cycle $C_{3}$ (see Fig. 1).

Fig. 2 and Fig. 3 present two blow-up graphs $G_{1}[H]$ and $G_{2}[H]$ of the graph $H$ presented in Fig. 1. In both cases we have the following values of the densities between clusters

$$
\begin{gathered}
d\left(A_{x}, A_{y}\right)=d\left(A_{y}, A_{z}\right)=\frac{3}{20}, \\
d\left(A_{x}, A_{v}\right)=\frac{3}{16}, \\
d\left(A_{y}, A_{v}\right)=\frac{1}{10} .
\end{gathered}
$$

Let us recall the definition of the multivariate matching polynomial of the graph. The polynomial is the useful tool for the proof of our results.

Definition 1. Let $H$ be a graph and let $x_{e}$ be the vector of variables $x_{e}, e \in E(H)$. We define the multivariate matching polynomial $F_{H}$ of the graph $H$ as follows

$$
F_{H}\left(\underline{x_{e}}, t\right)=\sum_{M \in \mathcal{M}}\left(\prod_{e \in M} x_{e}\right)(-t)^{|M|},
$$

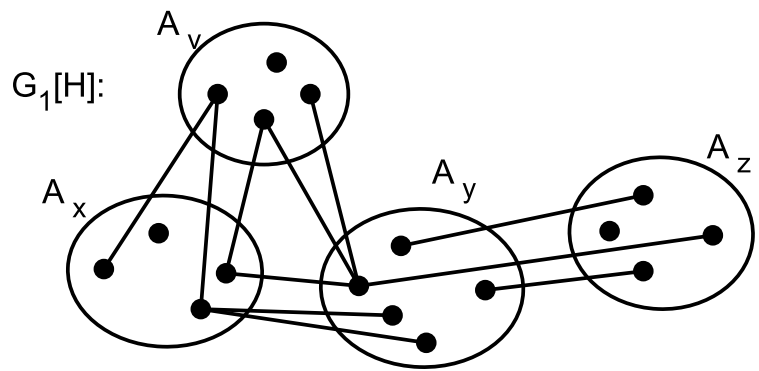

Fig. 2. An example of the blow-up graph $G[H]$ of the graph $H$ presented in Fig. 1 with a transversal $H$.

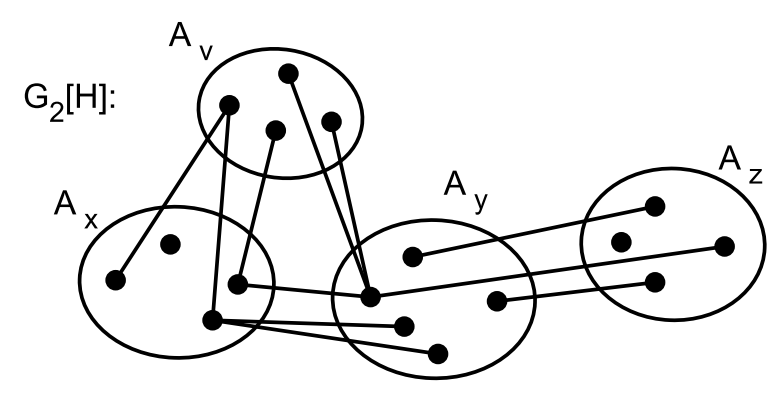

Fig. 3. An example of the blow-up graph $G[H]$ of the graph $H$ presented in Fig. 1 without a transversal $H$.

where the summation goes over all matchings of the graph $H$, including the empty matching.

Fig. 4 and Fig. 5 present the paths $P_{2}, P_{4}$ and the unicycle graph $H \in \mathcal{U}_{6,3}$ with variables $x_{e}$ assigned to each edge.
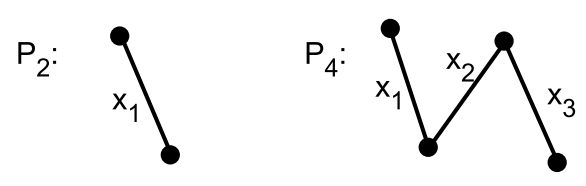

Fig. 4. Paths $P_{2}$ and $P_{4}$ with variables $x_{e}$ assigned to each edge.

$H$ :

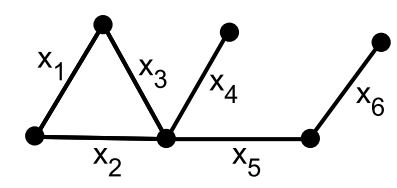

Fig. 5. Graph $H \in \mathcal{U}_{6,3}$ with variables $x_{e}$ assigned to each edge.

By definition of the multivariate matching polynomial we have

$$
\begin{gathered}
F_{P_{2}}\left(\underline{x_{e}}, s\right)=1-s x_{1}, \\
F_{P_{4}}\left(\underline{x_{e}}, s\right)=1-s\left(x_{1}+x_{2}+x_{3}\right)+s^{2} x_{1} x_{3}, \\
F_{H}\left(\underline{x_{e}}, s\right)=1-s\left(x_{1}+x_{2}+x_{3}+x_{4}+x_{5}+x_{6}\right)+ \\
s^{2}\left(x_{1} x_{4}+x_{1} x_{5}+x_{1} x_{6}+x_{2} x_{6}+x_{3} x_{6}+x_{4} x_{6}\right)-s^{3} x_{1} x_{4} x_{6} .
\end{gathered}
$$




\section{Some RESUlts FOR THE HOMOGENEOUS CASE}

For the completness of this paper we present some results for the homogeneous Turán density problem in this section. For this case Nagy [9] presented the following lower and upper bounds for the critical density $d_{\text {crit }}$.

Theorem 1 (Nagy [9]). For a graph $H$ we have

$$
\left(1-\frac{1}{\Delta(H)}\right) \leq d_{\text {crit }}(H) \leq\left(1-\frac{1}{\Delta^{2}(H)}\right)
$$

where $\Delta(H)$ is the maximal degree of $H$.

Then Csikvári and Nagy [5] improved the upper bound.

Theorem 2 (Csikvári and Nagy [5]). Let $\Delta(H)$ be the largest degree of the graph $H$. Then we have

$$
d_{\text {crit }}(H) \leq 1-\frac{1}{e(2 \Delta(H)-1)}
$$

where $e$ is the base of the natural logarithm.

Now let us recall the definition of the matching polynomial of the graph.

Definition 2. Let $H$ be a weighted graph with constant weight function $w(e)=1$ for all edges $e \in E(H)$. Then the matching polynomial is defined as

$$
M(H, t)=\sum_{k=0}^{n / 2}(-1)^{k} m_{k}(H) t^{n-2 k},
$$

where $m_{k}(H)$ denotes the number of $k$ independent edges in the graph $H$.

Using this polynomial Csikvári and Nagy [5] stated the upper bound for the critical density as in Theorem 3 .

Theorem 3 (Csikvári and Nagy [5]). Let $\Delta(H)$ be the largest vertex degree in the graph $H$ and let $t(H)$ be the largest root of the matching polynomial. Then we have

$$
d_{\text {crit }}(H) \leq 1-\frac{1}{(t(H))^{2}} .
$$

In particular,

$$
d_{\text {crit }}(H)<1-\frac{1}{4(\Delta(H)-1)} .
$$

What is more Nagy [9] showed the exact value of the critical density for trees.

Theorem 4 (Nagy [9]). Let $T$ be a tree. Then we have

$$
d_{\text {crit }}(T)=1-\frac{1}{\lambda_{\max }^{2}(T)},
$$

where $\lambda_{\max }(T)$ is the maximum eigenvalue of the adjacency matrix of the tree.

Furthermore, Nagy [9] showed that for the cycle of order $n$ and for the path of order $n+1$ the critical densities are equal.

Theorem 5 (Nagy [9]). Let $C_{n}$ be a cycle on $n$ vertices and $P_{n+1}$ be a path on $n+1$ vertices. Then we have

$$
d_{\text {crit }}\left(C_{n}\right)=d_{\text {crit }}\left(P_{n+1}\right)=1-\frac{1}{4 \cos ^{2} \frac{\pi}{n+2}} .
$$

We formulate the followig open problem.

Open problem: count the critical density $d_{\text {crit }}(H)$ for $H \in$ $\mathcal{U}_{n, p}, p \geq 3$.

\section{INHOMOGENEOUS CASE: UNICYCLIC GRAPHS WITH THE CYCLE $C_{3}$}

In this section we study the inhomogeneous case when graph $H \in \mathcal{U}_{n, 3}$, e.i. $H$ is unicyclic with the cycle $C_{3}$ and for each edge $e \in E(H)$ the edge density $\gamma_{e}$ is given. We extend some results presented in [5], where authors studied the inhomogeneous case for trees and proved the following theorem.

Theorem 6. (Csikvári, Nagy [5]) Let T be a tree of order $n$ and let $v$ be a leaf of $T$. Assume that for each edge of $T$ a density $\gamma_{e}=1-r_{e}$ is given. Let $T^{\prime}$ be a tree obtained from $T$ by deleting the leaf $v$ and the edge uv, where $u$ is the unique neighbour of $v$. Let the edge densities $\gamma_{e}^{\prime}$ in $T^{\prime}$ be defined as follows

$$
\gamma_{e}^{\prime}= \begin{cases}\gamma_{e}=1-r_{e}, & \text { if } e \text { is not incident to } u, \\ 1-\frac{r_{e}}{1-r_{u v}}, & \text { if } e \text { is incident to } u .\end{cases}
$$

Then the set of densities $\left\{\gamma_{e}\right\}_{e \in E(T)}$ ensures the existence of the factor $T$ if and only if all $\gamma_{e}^{\prime} \in(0,1]$ and the set of densities $\left\{\gamma_{e}^{\prime}\right\}_{e \in E\left(T^{\prime}\right)}$ ensures the existence of the factor $T^{\prime}$.

Theorem 6 provides authors of [5] with an efficient algorithm to decide whether a given set of edge densities in tree ensures the existence of a transversal or does not ensure. Their algorithm is presented below as Algorithm $T$ for the completeness of our paper.

We extend the algorithm (Algorithm $T$ ) to the family of unicyclic graphs with the cycle $C_{3}$. The new algorithm (Algorithm $\mathcal{U}_{n, 3}$ ) is based on the following Theorem 7 proved below by an extension of the method discovered in [5].

Theorem 7. Let $H \in \mathcal{U}_{n, 3}$ be a unicyclic graph of order $n$ with the cycle $C_{3}$ and assume that for each edge $e \in E(H)$ a density $\gamma_{e}=1-r_{e}$ is given. If the order of $H$ is greater then 3 , let $v$ be a leaf of $H$ and $u$ be the unique neighbour of $v$, then let $H^{\prime}$ be a graph obtained from $H$ by deleting the leaf $v$ and an edge uv. Let the densities $\gamma_{e}^{\prime}$ in $H^{\prime}$ be defined as follows

$$
\gamma_{e}^{\prime}= \begin{cases}\gamma_{e}=1-r_{e}, & \text { if } e \text { is not incident to } u, \\ 1-\frac{r_{e}}{1-r_{u v}}, & \text { if } e \text { is incident to } u .\end{cases}
$$

If the order of $H$ is equal to 3 (i.e., $H$ is isomorphic to $C_{3}$ with $V(H)=\{a, b, c\})$, then let $H^{\prime}$ be a graph obtained from $H$ by deleting the vertex $a$ and edges $a b$ and $a c . H^{\prime}$ is a path $P_{b c}$. Let the density $\gamma_{b c}^{\prime}$ in $H^{\prime}$ be defined as follows

$$
\gamma_{b c}^{\prime}=1-\frac{r_{b c}}{\left(1-r_{a b}\right)\left(1-r_{a c}\right)} \text {. }
$$




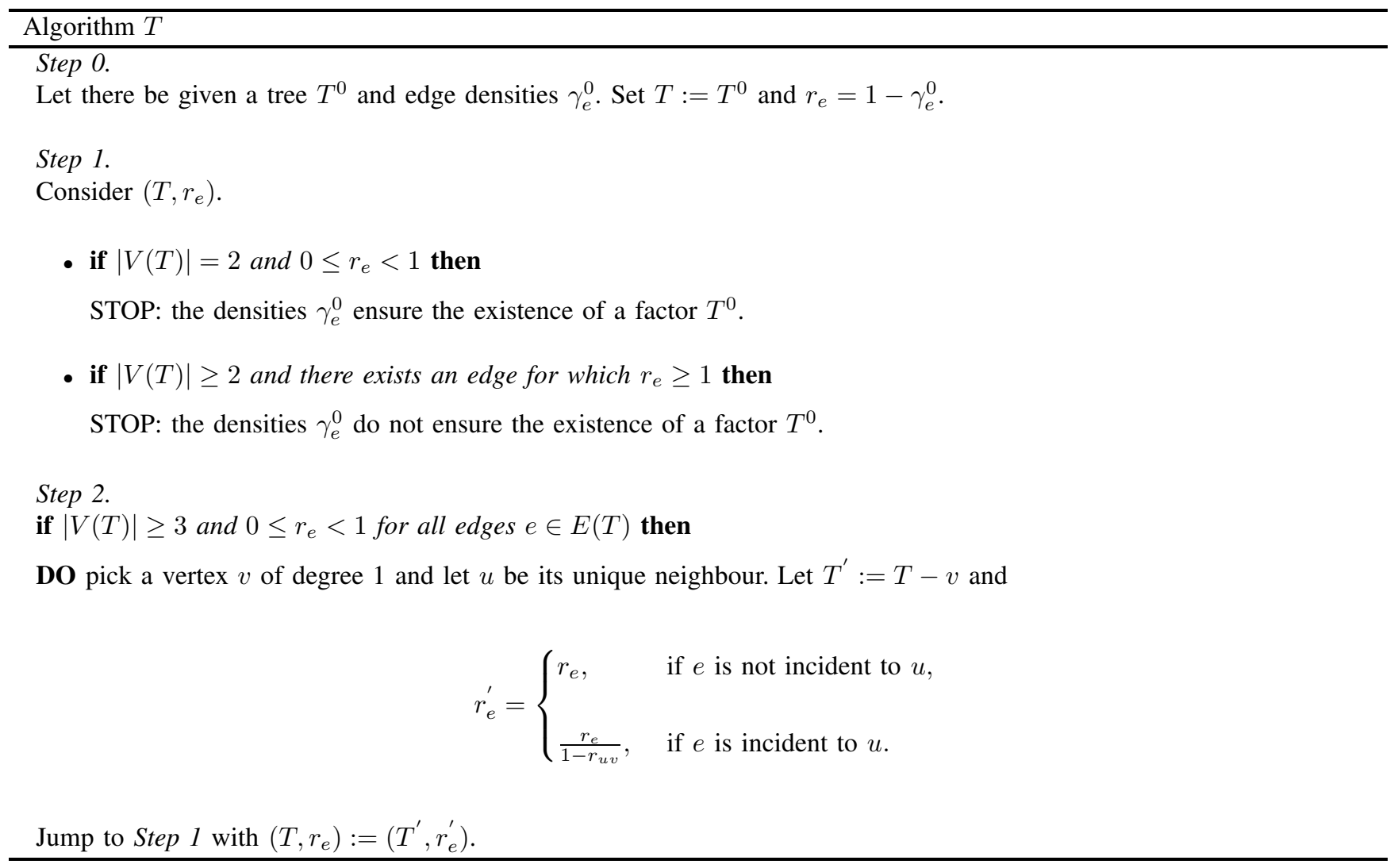

Then the set of densities $\left\{\gamma_{e}\right\}_{e \in E(H)}$ ensures the existence of the factor $H$ if and only if all $\gamma_{e}^{\prime} \in(0,1]$ and the set of densities $\left\{\gamma_{e}^{\prime}\right\}_{e \in E\left(H^{\prime}\right)}$ ensures the existence of the factor $H^{\prime}$.

Proof. Let $H \in \mathcal{U}_{n, 3}$ and let the set of densities $\gamma_{e}=1-r_{e}$ be given for each $e \in E(H)$. First we prove the following statement: if all $\gamma_{e}^{\prime}$ are indeed densities and they ensure the existence of a factor $H^{\prime}$, then the original densities $\gamma_{e}$ ensure the existence of a factor $H$.

Let $G[H]$ be a blow-up of the graph $H$ such that the density between $A_{i}$ and $A_{j}$ is at least $\gamma_{i j}$, where $A_{i}$ is a cluster of the vertex $i \in V(H)$. We show that it contains a factor $H$.

Let us consider a graph $H \in \mathcal{U}_{n, 3}$ with $n>3$ vertices.

Let $v, u \in V(H)$, where $v$ is a leaf of $H$ and $u \in N_{H}(v)$.

Define $R_{v, u}$ as the subset of $A_{u}$ in the following way (see Fig. 6).

$R_{v, u}=\left\{x \in A_{u} \mid x\right.$ is incident to some edge between

$$
\left.A_{u} \text { and } A_{v}\right\} \text {. }
$$

Note that

$$
\left|R_{v, u}\right|\left|A_{v}\right| \geq e\left(R_{v, u}, A_{v}\right)=\gamma_{u v}\left|A_{u}\right|\left|A_{v}\right| .
$$

Hence

$$
\left|R_{v, u}\right| \geq \gamma_{u v}\left|A_{u}\right| \text {. }
$$

Now we show the lower bound for the number of edges incident to $R_{v, u}$. Let $k \in N_{H}(u)$. By the inclusion - exclusion

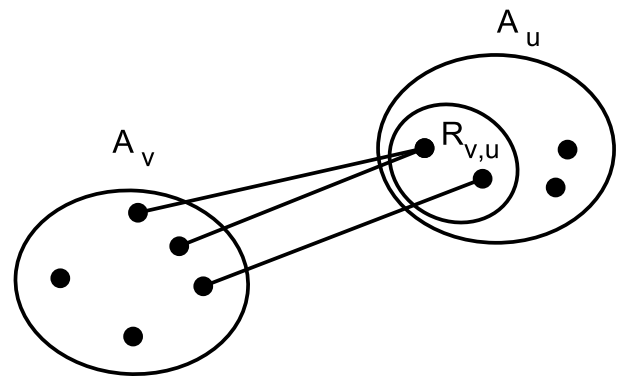

Fig. 6. Clusters $A_{v}$ and $A_{u}$ with the set $R_{v, u}$.

formula we count the lower bound for the number of edges between $R_{v, u}$ and $A_{k}$ as follows.

$$
\begin{gathered}
e\left(R_{v, u}, A_{k}\right) \geq e\left(A_{u}, A_{k}\right)-\left(\left|A_{u}\right|-\left|R_{v, u}\right|\right)\left|A_{k}\right|= \\
\left|R_{v, u}\right|\left|A_{k}\right|+\left(\gamma_{k u}-1\right)\left|A_{k}\right|\left|A_{u}\right| \geq \\
\left|R_{v, u}\right|\left|A_{k}\right|+\left(\gamma_{k u}-1\right) \frac{1}{\gamma_{u v}}\left|R_{v, u}\right|\left|A_{k}\right|= \\
\left(1-\frac{r_{k u}}{1-r_{u v}}\right)\left|R_{v, u}\right|\left|A_{k}\right|=\gamma_{k u}^{\prime}\left|R_{v, u}\right|\left|A_{k}\right| .
\end{gathered}
$$

Now, by deleting the vertex set $A_{v}$ and $A_{u} \backslash R_{v, u}$ from $G[H]$, we obtain a graph which is a blow-up of $H^{\prime}$ with edge densities ensuring the existence of the factor $H^{\prime}$. 


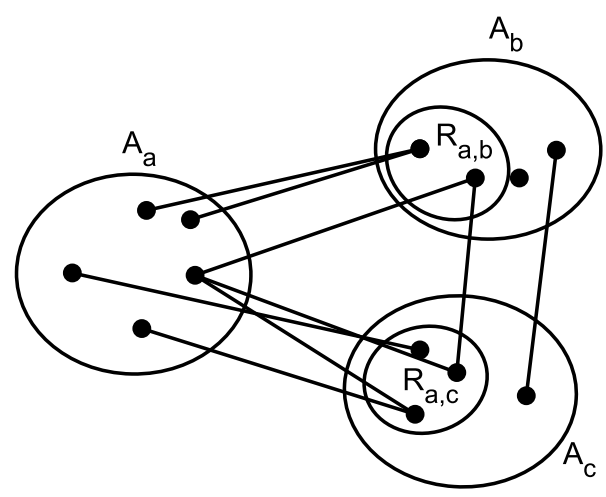

Fig. 7. Clusters $A_{a}, A_{b}$ and $A_{c}$ with the sets $R_{a, b}$ and $R_{a, c}$.

Moreover, by the definition of $R_{v, u}$ the factor $H^{\prime}$ can be extended to a factor $H$.

Now consider the situation when $n=3$ and graph $H$ is a cycle $C_{3}$ with the vertex set $\{a, b, c\}$. Let $A_{a}$ be a cluster of vertex $a$. Define sets $R_{a, b}$ and $R_{a, c}$ in the following way (see Fig. 7).

$R_{a, b}=\left\{x \in A_{b} \mid x\right.$ is incident to some edge between $A_{b}$ and $\left.A_{a}\right\}$,

$R_{a, c}=\left\{x \in A_{c} \mid x\right.$ is incident to some edge between $A_{c}$ and $\left.A_{a}\right\}$.

Note that

$$
\begin{aligned}
& \left|R_{b}\right|\left|A_{a}\right| \geq e\left(R_{b}, A_{a}\right)=\gamma_{a b}\left|A_{a}\right|\left|A_{b}\right|, \\
& \left|R_{c}\right|\left|A_{a}\right| \geq e\left(R_{c}, A_{a}\right)=\gamma_{a c}\left|A_{a}\right|\left|A_{c}\right| .
\end{aligned}
$$

Hence we have the following lower bounds for the cardinalities of $R_{a, b}$ and $R_{a, c}$

$$
\left|R_{a, b}\right| \geq \gamma_{a b}\left|A_{b}\right|
$$

and

$$
\left|R_{a, c}\right| \geq \gamma_{a c}\left|A_{c}\right|
$$

Next we show how many edges are incident to $R_{a, b}$ and $R_{a, c}$. Using the inclusion - exclusion formula we count the lower bound for the number of edges between $R_{a, b}$ and $R_{a, c}$

$$
\begin{gathered}
e\left(R_{a, b}, R_{a, c}\right) \geq e\left(A_{b}, A_{a}\right)-\left(\left|A_{b}\right|-\left|R_{a, b}\right|\right)\left|A_{c}\right|- \\
\left(\left|A_{c}\right|-\left|R_{a, c}\right|\right)\left|A_{b}\right|+\left(\left|A_{b}\right|-\left|R_{a, b}\right|\right)\left(\left|A_{c}\right|-\left|R_{a, c}\right|\right)= \\
\left|R_{a, b}\right|\left|R_{a, c}\right|+\left(\gamma_{b c}-1\right)\left|A_{b}\right|\left|A_{a}\right| \geq \\
\left|R_{a, b}\right|\left|R_{a, c}\right|+\left(\gamma_{b c}-1\right) \frac{1}{\gamma_{a b}} \frac{1}{\gamma_{a c}}\left|R_{a, b}\right|\left|R_{a, c}\right|= \\
\left(1-\frac{r_{b c}}{\left(1-r_{a b}\right)\left(1-r_{a c}\right)}\right)\left|R_{a, b}\right|\left|R_{a, c}\right|=\gamma_{b c}^{\prime}\left|R_{a, b}\right|\left|R_{a, c}\right| .
\end{gathered}
$$

Now, by deleting the vertex sets $A_{a}, A_{b} \backslash R_{a, b}$ and $A_{c} \backslash R_{a, c}$ from $G\left[C_{3}\right]$, we obtain a graph which is a blow-up of $C_{3}^{\prime}=P_{2}$, $V\left(P_{2}\right)=\{b, c\}$, with edge densities ensuring the existence of

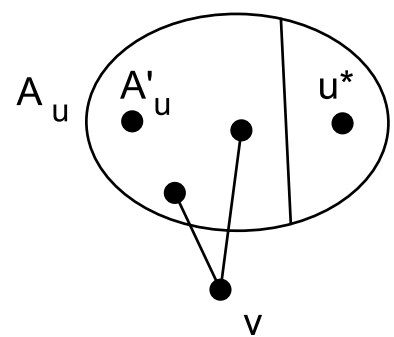

Fig. 8. We assume that $G^{\prime}\left[H^{\prime}\right]$ is without transversal $H^{\prime}$. The construction of the blow-up graph $G[H]$ without transversal $H$ for the case where $v$ is a leaf in $H$ and $H^{\prime}=H-v$. The cluster $A_{u}^{\prime}$ is in $G^{\prime}\left[H^{\prime}\right]$. Let $A_{u}=A_{u}^{\prime} \cup u^{*}$ and $A_{v}=\{v\}$ be clusters in $G[H]$.

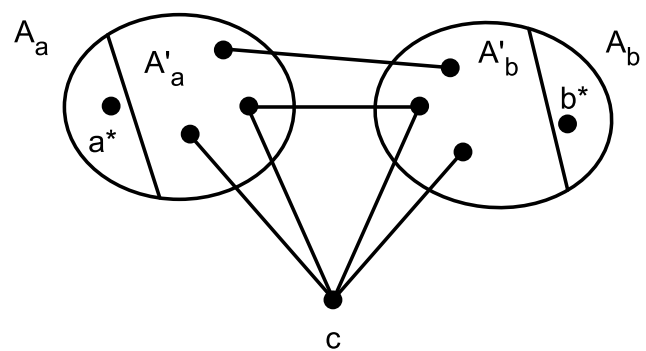

Fig. 9. We assume that $G^{\prime}\left[H^{\prime}\right]$ is without transversal $H^{\prime}$. The construction of the blow-up graph $G[H]$ without transversal $H$ for the case where $c$ is a vertex of $C_{3}, V\left(C_{3}\right)=\{a, b, c\}$ in $H$ and $H^{\prime}=H-c$. The clusters $A_{a}^{\prime}$ and $A_{b}^{\prime}$ are in $G^{\prime}\left[H^{\prime}\right]$. Let $A_{a}=\left\{a^{*}\right\} \cup A_{a}^{\prime}, A_{b}=\left\{b^{*}\right\} \cup A_{b}^{\prime}$ and $A_{c}=\{c\}$ be clusters in $G[H]$.

the factor $P_{2}$. Moreover, by the definition of $R_{a, b}$ and $R_{a, c}$ the factor $P_{2}$ can be extended to a factor $C_{3}$.

Note that if

$$
\gamma_{k u}^{\prime}<0
$$

then

$$
\gamma_{k u}+\gamma_{u v}<1
$$

So there exists a construction which does not induce the path $P_{3}$ with the consecutive vertices $k, u, v$, where $i \in A_{i}(i \in$ $\{k, u, v\})$ in this case. Therefore, if some $\gamma_{k u}^{\prime}<0$ then there exists a construction for a blow-up graph of $H$ without a factor of $H$.

Next assume that all the $\gamma_{e}^{\prime}$ are proper densities, but there is a construction of a blow-up graph, say $G^{\prime}\left[H^{\prime}\right]$, with edge densities at least $\gamma_{e}^{\prime}$, but which does not induce a factor $H^{\prime}$. Thus we construct a blow-up $G[H]$ of the graph $H$ not inducing $H$. We consider two possible cases. First let the picked vertex $v$ be a leaf in $H$ and $H^{\prime}=H-v$. Then set $A_{u}=\left\{u^{*}\right\} \cup A_{u}^{\prime}$ and $A_{v}=\{v\}$. We connect $v$ to all elements of $A_{u}^{\prime}$ but do not connect to $u^{*}$ without changing densities in $G^{\prime}\left[H^{\prime}\right]$ and with density $\gamma_{v u}$ (see Fig. 8).

Now let $H^{\prime}=H-c$, where $c$ is a vertex of $C_{3}, V\left(C_{3}\right)=$ $\{a, b, c\}$. Then set $A_{a}=\left\{a^{*}\right\} \cup A_{a}^{\prime}, A_{b}=\left\{b^{*}\right\} \cup A_{b}^{\prime}$ and $A_{c}=\{c\}$. We connect $c$ to all elements of $A_{a}^{\prime}$ and $A_{b}^{\prime}$ but do not connect to $a^{*}$ and $b^{*}$ without changing densities in $G^{\prime}\left[H^{\prime}\right]$ and with densities $\gamma_{c a}$ and $\gamma_{c b}$ (see Fig. 9). 
Theorem 7 provides us with the algorithm (Algorithm $\mathcal{U}_{n, 3}$ ) to decide whether a given set of edge densities ensures the existence of a transversal $H$ in a blow-up graph $G[H]$ or does not ensure, where $H \in \mathcal{U}_{n, 3}$.

For further considerations recall some results presented in papers [3] and [5]. First lemma gives condition on edge densities in the triangle $C_{3}$ which allows us to check if these densities ensure existing of $C_{3}$ in a blow-up graph $G\left[C_{3}\right]$. The second results gives condition on existing graph $H$ as a factor in a blow-up graph $G[H]$ in terms of the multivariate matching polynomial $F_{H}$.

Lemma 1. (Bondy, et al. [3]) Let $\alpha, \beta$, $\gamma$ be the edge densities between the clusters of a blow-up graph of the triangle - a cycle $C_{3}$. If

$$
\alpha \beta+\gamma>1, \beta \gamma+\alpha>1, \gamma \alpha+\beta>1,
$$

then the blow-up graph contains a triangle as a transversal.

Theorem 8. (Csikvári, Nagy [5]) Assume that for the graph $H$ we have

$$
F_{H}\left(\underline{r_{e}}, t\right)>0
$$

for all $t \in[0,1]$ and some vector $\underline{r}_{e}$ of weights, where $r_{e} \in$ $[0,1]$ for each edge $e \in E(H)$. Then the densities $\gamma_{e}=1-r_{e}$ ensure the existence $H$ as a transversal.

Let $H:=C_{3}$ with vertices $a, b, c$ and edge densities $\gamma_{e}=$ $1-r_{e}$, where $e \in\{a b, b c, a c\}$. Assume that all $r_{e} \in[0,1)$ and run the Algorithm $\mathcal{U}_{n, 3}$ by deleting vertex $a$ from the graph $C_{3}$ with edges incident to it (means $a b$ and $a c$ ). As a result we get a graph $H^{\prime}$ as a path $P_{2}=b c$ with edge density

$$
\gamma_{b c}^{\prime}=1-r_{b c}^{\prime}=1-\frac{r_{b c}}{\left(1-r_{a b}\right)\left(1-r_{a c}\right)} \text {. }
$$

For $H^{\prime}$ we have

$$
F_{H^{\prime}}\left(\underline{r_{e}}, t\right)=1-t r_{b c}^{\prime} .
$$

By Theorem 8 we need

$$
F_{H^{\prime}}\left(\underline{r_{e}}, t\right)>0
$$

for $t \in[0,1]$.

Hence

$$
\begin{gathered}
\frac{1}{r_{b c}^{\prime}}>1, \\
\left(1-r_{a b}\right)\left(1-r_{a c}\right)-r_{b c}>0
\end{gathered}
$$

and

$$
\gamma_{a b} \gamma_{a c}+\gamma_{b c}>1
$$

Similar inequalities are received when, instead of a vertex $a$, we delete in Algorithm $\mathcal{U}_{n, 3}$ vertex $b$ or $c$. As we can see we have a result presented in Proposition 1 consensual with Lemma 1.

Proposition 1. Let $a, b, c$ be vertices in a triangle $C_{3}$. Assume that $\gamma_{e}=1-r_{e}$ be an edge density assigned to each edge $e \in E\left(C_{3}\right)$, where $E\left(C_{3}\right)=\{a b, a c, b c\}$. If

$$
\frac{r_{a b}}{\left(1-r_{a c}\right)\left(1-r_{b c}\right)}<1, \frac{r_{a c}}{\left(1-r_{a b}\right)\left(1-r_{b c}\right)}<1
$$

$$
\text { and } \frac{r_{b c}}{\left(1-r_{a b}\right)\left(1-r_{a c}\right)}<1 \text {, }
$$

then the set of densities $\left\{\gamma_{e}\right\}_{e \in E\left(C_{3}\right)}$ ensures existence of a transversal $C_{3}$ in a blow-up graph $G\left[C_{3}\right]$.

By running Algorithm $\mathcal{U}_{n, 3}$ on some unicyclic graph $H \in$ $\mathcal{U}_{n, 3}$ with $\gamma_{e}=1-t r_{e}$ and using the multivariate matching polynomial $F_{H}\left(\underline{r_{e}}, s\right)$ we can prove the following lemma.

Lemma 2. Let $H$ be a weighted unicyclic graph of order $n>2$ with the cycle $C_{3}$. Let $\gamma_{e}=1-t_{e}$ be densities assigned to each edge $e \in E(H)$, where $r_{e} \in[0,1)$. Assume that after running Algorithm $\mathcal{U}_{n, 3}$ we get a cycle $C_{3}$ with

$$
F_{C_{3}}\left(\underline{r_{e}}, t\right)=0 \text {, }
$$

then $t$ is a root of the multivariate matching polynomial $F_{H}\left(\underline{r_{e}}, s\right)$ of the graph $H$.

Proposition 2. Let $H$ be a weighted unicyclic graph of order $n>2$ with the cycle $C_{3}$. Let $\gamma_{e}=1-t r_{e}$ be the densities assigned to each edge $e \in E(H)$. Assume that after running Algorithm $\mathcal{U}_{n, 3}$ we get a cycle $C_{3}$ with the vertex set $V\left(C_{3}\right)=$ $\{a, b, c\}$ and with $F_{C_{3}}\left(\underline{r}_{e}, t\right)=0$ and, after restart Algorithm $\mathcal{U}_{n, 3}$, we get a path $P_{2}$ (by deleting the vertex a and edges $a b, a c)$, then

$$
F_{P_{2}}\left(\underline{r_{e}^{\prime}}, s\right)=\frac{t^{2} r_{a b} r_{a c}+t r_{b c}}{\left(1-t r_{a b}\right)\left(1-t r_{a c}\right)}-s \frac{t r_{b c}}{\left(1-t r_{a b}\right)\left(1-t r_{a c}\right)} .
$$

Proof. Assume that after running Algorithm $\mathcal{U}_{n, 3}$ we get a cycle $C_{3}$ with edge densities $\gamma_{e}=1-t r_{e}$. Let $V\left(C_{3}\right)=$ $\{a, b, c\}$ and $r_{a b}, r_{a c}, r_{b c} \in[0,1)$. The multivariate matching polynomial

$$
F_{C_{3}}\left(\underline{r_{e}}, s\right)=1-s\left(r_{a b}+r_{a c}+r_{b c}\right)
$$

has exactly one root

$$
t=\frac{1}{\left(r_{a b}+r_{a c}+r_{b c}\right)} .
$$

By deleting vertex $a$ from the cycle $C_{3}$ with the edges $e_{a b}=$ $a b$ and $e_{a c}=a c$ we obtain a path $P_{2}=b c$. By Theorem 7 we get that

$$
F_{P_{2}}\left(\underline{r_{e}^{\prime}}, s\right)=1-s r_{b c}^{\prime}=1-s \frac{t r_{b c}}{\left(1-t r_{a b}\right)\left(1-t r_{a c}\right)} .
$$

By multiplying both sides by

$$
\left(1-t r_{a b}\right)\left(1-t r_{a c}\right)
$$

we have

$$
\begin{gathered}
\left(1-t r_{a b}\right)\left(1-t r_{a c}\right) F_{P_{2}}\left(\underline{r_{e}^{\prime}}, s\right)= \\
\left(1-t r_{a b}\right)\left(1-t r_{a c}\right)-s t r_{b c}= \\
1-t\left(r_{a b}+r_{a c}+r_{b c}\right)+t r_{b} c+t^{2} r_{a b} r_{a c}-s t r_{b c} .
\end{gathered}
$$

So

$$
\begin{gathered}
\left(1-t r_{a b}\right)\left(1-t r_{a c}\right) F_{P_{2}}\left(\underline{r_{e}}, s\right)-t^{2} r_{a b} r_{a c}-t r_{b c}+s t r_{b c}= \\
F_{C_{3}}\left(\underline{r_{e}}, t\right)=0 .
\end{gathered}
$$




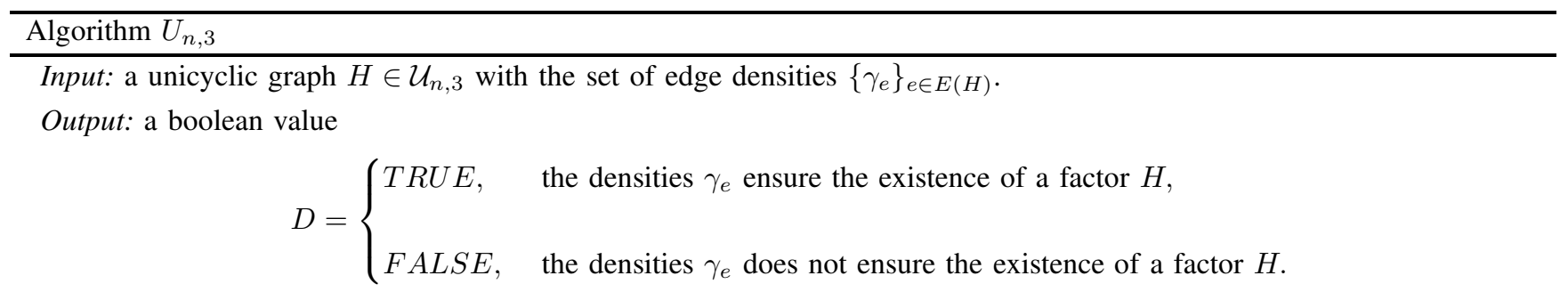

Consider a weighted graph $\left(H, r_{e}\right)$, where $r_{e}=1-\gamma_{e}$.

Step 1.

- if $|V(H)|=2$ (means $H$ is a path $P_{2}$ ) and $0 \leq r_{e}<1$ then

STOP: $D:=$ TRUE.

- if $|V(H)| \geq 2$ and there exists an edge for which $r_{e} \geq 1$ then

STOP: $D:=$ FALSE.

Step 2.

- if $|V(H)|=3$ (means $H$ is a cycle $C_{3}$ ) and $0 \leq r_{e}<1$ for all edges $e \in E(H)$ then pick a vertex $c$ of the graph $H$ and let $a, b$ be its neighbours. Let $H^{\prime}:=H-c$ and

$$
r_{a b}^{\prime}=\frac{r_{a b}}{\left(1-r_{a c}\right)\left(1-r_{b c}\right)}
$$

- if $|V(H)|>3$ and $0 \leq r_{e}<1$ for all edges $e \in E(H)$ then

pick a vertex $v$ of degree 1 and let $u$ be its unique neighbour. Let $H^{\prime}:=H-v$ and

$$
r_{e}^{\prime}= \begin{cases}r_{e}, & \text { if } e \text { is not incident to } u, \\ \frac{r_{e}}{1-r_{u v}}, & \text { if } e \text { is incident to } u .\end{cases}
$$

Go to Step 1 with $\left(H, r_{e}\right):=\left(H^{\prime}, r_{e}^{\prime}\right)$.

Hence

$$
F_{P_{2}}\left(\underline{r}_{e}^{\prime}, s\right)=\frac{t^{2} r_{a b} r_{a c}+t r_{b c}}{\left(1-t r_{a b}\right)\left(1-t r_{a c}\right)}-s \frac{t r_{b c}}{\left(1-t r_{a b}\right)\left(1-t r_{a c}\right)}
$$

By the definition of $F_{P_{2}}\left(\underline{r_{e}^{\prime}}, s\right)$ we have

and

$$
\frac{t^{2} r_{a b} r_{a c}+t r_{b c}}{\left(1-t r_{a b}\right)\left(1-t r_{a c}\right)}=1
$$

$$
t\left(r_{a c}+r_{a b}+r_{b c}\right)=1
$$

Note that if

$$
\gamma_{b c}^{\prime}=1-\frac{t r_{b c}}{\left(1-t r_{a b}\right)\left(1-t r_{a c}\right)}=0
$$

then

$$
t r_{b c}=\left(1-t r_{a b}\right)\left(1-t r_{a c}\right)
$$

and

$$
\begin{gathered}
\frac{t^{2} r_{a b} r_{a c}}{\left(1-t r_{a b}\right)\left(1-t r_{a c}\right)}=0 \\
t r_{a b} t r_{a c}=0
\end{gathered}
$$

Therefore,

$$
t\left(r_{a c}+r_{a b}+r_{b c}\right)=1+t r_{a b} t r_{a c}=1 .
$$

So $t$ is the root of $F_{C_{3}}\left(\underline{r_{e}}, t\right)$. 
From above consideration we deduce that Algorithm $\mathcal{U}_{n, 3}$ works correctly with time complexity at most $\mathcal{O}\left(n^{2}\right)$. Algorithm $\mathcal{U}_{n, 3}$ can be implemented in such a way that a vertex of the subgraph $C_{3}$ be considered (picked) in the last step of the algorithm.

\section{CONCLUSION}

We have presented Algorithm $\mathcal{U}_{n, 3}$ for testing whether the unicyclic graph $H \in \mathcal{U}_{n, 3}$ with the set of edge densities $\left\{\gamma_{e}\right\}_{e \in E(H)}$ is a factor of a blow-up graph $G[H]$. Precisely, we have the answer whether the edge densities ensure the existence of the factor or do not ensure. In future work we will study the density Turán problem for an arbitrary graph of the family $\mathcal{U}_{n, p}, p \geq 4$, and for other families of graphs. Moreover, we wish to construct efficient algorithms for testing the existence of blow-up graphs with factors of the families.

Open problem: Look for the density Turán problem algorithm for families of connected graphs with blocks (i.e., 2-connected components) isomorphic to cycles and/or $P_{2}$.

\section{REFERENCES}

[1] R. Baber, J.R. Johnson and J. Talbot, The minimal density of triangles in tripartite graphs, LMS J. Comput. Math., 13 (2010), 388-413, http://dx.doi.org/10.1112/S1461157009000436.

[2] B. Bollobás, Extremal Graph Theory, Academic Press (1978).

[3] A. Bondy, J. Shen, S. Thomassé and C. Thomassen, Density Conditions for triangles in multipartite graphs, Combinatorica, 26 (2006), http://dx.doi.org/10.1007/s00493-006-0009-y.

[4] W.G Brown, P. Erdös and M. Simonovits, Extremal problems for directed graphs, Journal of Combinatorial Theory, Series B 15 (1) (1973), 77-93, http://dx.doi.org/10.1016/0095-8956(73)90034-8.

[5] P. Csikvári and Z. L. Nagy, The density Turán Problem, Combinatorics, Probability and Computing, 21 (2012), 531-553, http://dx.doi.org/10.1017/S0963548312000016.

[6] Z. Füredi, Turán type problems, Survey in Combinatorics Vol. 166 of London Math. Soc. Lecture Notes (A.D. Keedwell, ed.) (1991), 253-300, http://dx.doi.org/10.1017/cbo9780511666216.010.

[7] C.D. Godsil and G. Royle, Algebraic Graph Theory, Springer (2001), http://dx.doi.org/10.1007/978-1-4613-0163-9.

[8] G. Jin, Complete subgraphs of $r$-partite graphs, Combin. Probab. Comput., 1 (1992), 241-250, http://dx.doi.org/10.1017/s0963548300000274.

[9] Z.L. Nagy, A multipartite version of the Turán problem - density conditions and eigenvalues, The Electronic Journal of Combinatorics, 18 (2011), \# P46.

[10] P. Turán, On an extremal problem in graph theory, Mat. Fiz. Lapok, 48 (1941), 436-452.

[11] R. Yuster, Independent transversal in $r$-partite graphs, Discrete Math., 176 (1997), 255-261, http://dx.doi.org/10.1016/s0012-365x(96)00300-7. 